\title{
Effective and Efficient ways of Hybridizing GA with various methods while reviewing a wide variety of Hybrid Genetic Approaches.
}

\author{
$21^{\text {st }}$ April, 2013
}

\author{
Nafisa Maqbool ${ }^{1}$,Dept. of \\ Industrial \& Manufacturing System \\ Engineering, School of Mechanical \\ Science \& EngineeringHuazhong \\ UniversityofScience\&Technology,Wuha \\ n,Hubei,430074,China.nafia k9@live.co \\ $\underline{\mathrm{m}}$.
}

\author{
Chaoyong \\ Zhang Associate \\ Professor and supervisorDept. of Industrial \\ \& Manufacturing System \\ Engineering, ,Huazhong University of \\ Science \& Technology, Wuhan, Hubei, \\ 430074.China.Corresponding author: \\ zcyhust@mail.hust.edu.cn
}

\author{
Mudabbir Baddir ${ }^{2} \cdot \mathrm{PhD}$ and \\ research assistant, Kochi University \\ of Technology, Japan. \\ mbbhutto@hotmail.com
}

\begin{abstract}
Hybrid genetic algorithms significant interest over the decade are increasingly used to resolve real-world problems. Genetic algorithm's ability to incorporate various techniques within its framework to produce a hybrid that secures the best from the blend. In this paper, different forms of integrations between genetic algorithms and various search and optimization techniques/methods will be focused on. This dissertation also aims to observe issues that acquire our consideration when designing a hybrid genetic algorithm that uses another search method as searching tools. Different approaches for employing these searching tool information and various mechanisms that acquire attaining a balance between global genetic algorithm and search tools.
\end{abstract}

Referenced terms - local search, genetic algorithm, hybrid, population, fitness, algorithms, Lamarckian

\section{INTRODUCTION}

Genetic algorithm (GA) has a population-based search space and optimization methods that impersonator's the process of evolution naturally. The two main concepts of evolution are natural selection (NS) and genetic dynamics (GD) they are enthused by the development of methods. The basic principles of these techniques were first approached by Holland et al [1] which are described, as examples in many

-papers. The recital of any genetic algorithm, like global optimization algorithm, are governed by the mechanis $\mathrm{m}$ of balancing conflicting objectives, which exploit the paramount solution found till date and at the same time explore the search space for feasible solutions. The supremacy of genetic algorithms comes from the ability that combines both examination and utilization of an optimal way [1].

Although this optimality of exploitation may be hypothetically factual for a GA, there are glitches in practice. These arise because Holland et al presumed that population size is unlimited and that the fitness performance accurately reflected to the control of his solutions. The interactions between genes are very minor [2].

The difficulty of finding the feasible solutions in supreme regions accounts the GA operator's poor performances that make minor changes in the neighborhood of the current solutions [3]. Covering the full use of a local search tool in the GA could better perform the exploiting abilities of the searching algorithm without restricting its exploring performance [4]. When the right balances are between global exploration and the local exploitation competences than it achieves the algorithm could easily produce feasible solutions with accuracy [5].

A mixture of a GA and a local search method could rapidly increase the search to locate the exact global solutions required. In hybrid GA's, implementing a local search for the solution that are required by a GA for the most feasible space could increase union to the global optimum solution. The time consumed to achieve global optimality could further reduced when local search tools are used to speed up the locating of the most feasible search spaces also when locating the global optimum solution that starts within the 
basin of pull. The not so perfect choices of controlling parameters are other ways of limiting GA's in resolving real-world problems [6] because of the detrimental effects with the trade-offs between utilization and exploration. Upon these parameters algorithms could either succeeding to find almost optimum solution by any efficient way or simply fail to find any solution. Choosing the correct parameters are time-consuming. Therefore, the use of strike controlling parameters become contradicting to the evolution of GA [7].

For these reasons, various searching techniques need to be utilized for setting these values for the parameters while the search are still in progress. In this dissertation, hybrid genetic algorithms (HGA's) are thoroughly examined when putting in the different ways in which their roles for the search methods and GA are integrated. The dissertation plans to present a not to classified hybrid genetic algorith ms, but to highlight on the possibilities when combining the search method together as a framework of GA. For an architectural taxonomy of combinatorial me-metic algorithms the reader could refer to 8] [9] and [10], whereby meta-heuristics are classed basing on the designing of the search space and implantation space.

Gain an insight of the design issues of hybrid genetic algorithms (HGA's) while also reviewing the different mechanisms that have been utilized by authors when applying local search contributions within genetic search also having the various techniques that achieved a balance with exploration and utilization.

\section{The problem}

Problems could also waste the resources of hybrids that use clustering techniques in the genotype domain to reduce unnecessary local search, in contrast to the Lamarckian approach which has been shown to help alleviate this problem [11]. Hart et al. [12] pointed to the importance of considering the cost of learning, which has been ignored by most researchers when studying the impact of the Baldwinian strategy on the hybrid search by analyzing its performance based on the number of generations of the genetic algorith $\mathrm{m}$ only.
Joines et al. [13] found that using the pure Lamarckian approach (100\% Lamarckian) produced the best convergence speed to the best known solution when solving the cell formation problem. Espinoza et al. [15] used $75 \%$ partial Lamarckian as the optimal leaning strategy in their hybrid to optimize two continuous functions. El-Mihoub et al. [14] investigated the combined effect of probability of local search and leaning strategy on the hybrid performance and found that combing a low probability of local search with the pure Lamarckian learning strategy can improve the convergence speed without disrupting the schema processing. Ishibushi et al. [16] found that the 5\% partial Lamarckian worked well on the multi-objective $0 / 1$ knap-sack problem using a single population model, however, the $50 \%$ partial Lamarckian was the optimal choice using the is land model

The same technique has been used to solve the threematching problem [17], where an adaptive hybrid algorithm selects one operator from eight recombination and local search operators based on their current and past benefit-cost ratio. Espinoza et al. [18] used the change in coefficient of variation of the fitness function to determine whether the genetic algorithm is exploring new regions of the search space or exploiting the already visited regions.

Although the aim of combining a global genetic algorith $m$ and a local search method is to get the best out of the exploring ability of the former, and the efficiency of the latter in reaching local optima, the two methods can interact in a more complicated way than the one described above. Rosin et al. [19] argued that the mutation operator in a hybrid plays a different role than it does in a pure genetic algorith $\mathrm{m}$.

In this way, the global genetic algorithm decides on the individuals that should perform a local search and on its Duration SUMMARY In this paper, we have tried to put light on the effectiveness and efficiency of hybridizing GA with various techniques through 
reviewing some of the wide variety of hybrid genetic approaches.

However, for large and complicated search spaces, their role is limited to accelerating finding of the global optimum once the genetic algorithm isolates the most promising region and can be helpful in later stages of the search. Duration of Local Search Local search duration influences the balance between the global exploration of genetic algorithms and local refinement of the neighborhood search method in hybrid genetic algorith ms [20], [21].

Most of hybrid genetic algorithms that repair chromosomes to satisfy constrains are Lamarckian and the technique has been particularly effective in solving TSP [22]. Baldwinian Learning allows an individual's fitness to be improved by applying a local search, whereas the genotype remains unchanged.

Their experiments showed that the algorithm is more efficient than a pure genetic algorithm and is stable against a greater range of parameter settings than the standard staged hybrid genetic algorithm. Hacker et al. [23] proposed an approach that switches between global genetic and local search, based on the local topology of the search space

Modifying the parameters of the local search, such as the frequency of local search, the duration of local search, and the probability of local search can help the hybrid to strike the balance between the two search methods.

Frequency of LSThe number of continuous uninterrupted generations that a genetic algorithm performs before applying local search is usually referred to as the frequency of local search.

The authors concluded that this adaptation mechanis $\mathrm{m}$ allows the balance between the global genetic search and the local search to be adjusted according to the particularities of the search space, thus allowing significant improvements in the performance for different classes of problems.

\section{LS Potential Techni que}

The LS potential selection mechanism has been proposed by Land [24] to decide which individuals should perform the local search.

A hybrid with long local search duration will execute fewer generations of the genetic algorithm than a hybrid with shorter local duration, if both terminate after the same number of function evaluations. Leng [70] proposed the guided genetic algorithm which is a hybrid genetic system that borrows the concept of feature and penalties from the guided local search [25].

The effectiveness of adopting the pure Lamarckian approach, the pure Baldwinian approach, or any mixtu re of them in a hybrid is affected by the fitness landscape, the representations, the percentage of population performs local search and local search method used [15], [26], [13], [27], [28], [16].

In the Lamarckian approach, the local search method is used as a refinement genetic operator that modifies the genetic structure of an individual and places it back in the genetic population.

They concluded that utilizing local search could be helpful for s mall search spaces in the early stages of search due to their role in helping the genetic algorith $m$ to define the most promising regions of the search space.

In traditional hybrid genetic algorithms, a local search is applied to every individual in the population. The Lamarckian local search can disrupt the schema processing of the genetic algorith $m$ which may lead to premature convergence and produce an ineffective search. The exploring ability of the 
genetic algorithm can be further improved by utilizing local search to ensure fair representation of different regions of a searchA decrease in the values of the coefficient of variance indicates that the genetic algorithm has converged to a small area of the search space and the search process can therefore be made more efficient by switching to a local search

The basic idea of this approach ignores the role of local search in improving the sampling ability of the genetic algorithm. The first goal of the hybridization, which is the effectiveness of search, can be satisfied if a genetic algorithm and a local search method cooperate in the manner mentioned above.The ability of a genetic-local hybrid to solve hard problems quickly depends on the way of utilizing local search information and the mechanism of balancing genetic and local search. Due to their major impact on hybrid genetic performance, the discussion is concentrated on the strategies of utilizing local search information within a hybrid, and mechanisms that can be used to achieve a balance between exploration and exploitation.

However, in a hybrid algorithm to solve the static correction problem [29], the genetic search algorithm was allowed to continue uninterrupted for ten generations before applying a single iteration of waveform steepest ascent iteration to each individual in the population.

Different techniques, such as tuning, distributionbased [4], fitness based [4] techniques, and local search potential [24], have been proposed to decide on the optimal fraction of the population that should perform a local search. In the genetic-local hybrid, the main role of the genetic algorith $m$ is to explore the search space in order to either isolate the most promising regions of the search space, or, to hit the global optimum. Researchers have proposed different techniques to enable the hybrid to mix both methods wisely or at least to reduce the consequences of the improper use of the expensive local search. The self- adaptation technique has also been used to adapt the duration of local search in a hybrid through encoding the number of local iterations into chromosomes [30].

The self-adaptation techniques are reported to be successfully used to decide between different local search methods in solving the One Max problem, NK-Landscapes, and TSP [33]. Joines and Kay [11] combined evolutionary algorithms with random linkage and borrowed the concept of short memory from tabu search [32] to avoid performing unnecess ary local search on non-promising regions of the search space.

\section{Conclusion}

This can improve the ability of the genetic algorith $\mathrm{m}$ to direct the search to the most promising regions of the search space. When a GA has a global search method that is combined to a specific problem method such as the local searching method, the overall search capabilities are enhanced.

However, applying a local search to every individual in the population on costly function evaluations can waste resources without providing any more useful information.

\section{Acknowledgement}

The design of this paper was supported by the state key program of national natural science of china (grant number 51035001), National natural science foundation of china (grant number 5127519).

\section{Reference}

1. J.holland, adapation in natural and artificial systems, the university of Michigan, 1975

2. D.Beasley, D.R.bull. R, and R. Martin, an overview of genetic algorithm, part1 ,fundemaentals , university computing, volume 15 page 58-69, 1993. 
3. C.Reeves, genetic algorithm and neighborhood search in elovutionary computing science, TC.Fogarty, ED. Leeds, UK, springer-verlag, page $115-130,1994$

4. W.E.Hart, apadaptive global optimization with local search, doctorail dissertation, san deigo,university of California, 1994

5. F.G Lobo, D.E.goldberg, decision making hybrid genetic algorithm, IEEE,internation conference on evolutionary computation,Piscataway, usa,page 122-125, 1997

6. K.Deb, limitations of evolutionary compution, T.Back,D.B.fogel and Zmichalewicz,eds,IOP publishing and oxford university press, 1997

7. A.E.Eiben, R.Hinterd in and Z.Michalewicz, parameter control in evolutionary algorithm IEEE transactions on evolutionary computation vol 12 page 124-141,1999

8. N.Krasnogor and J.smith, a tutorial for competent memtic algorithm, model taxonomy and design is sues, IEEE transaction

9. P.Moscato,an evolutionary search optimization genetic algorithms and martial arts, towards memtic algorithm, California institute of technology,1989

10. E.Talbi, a taxanomy of hybrid metaheuristics, journal of heuristics, volume 8 page 541 564,2002

11. J.A.Joines and MGKay,hybrid genetic algorith $m$ and random linkage, in 2002 congress on evolutionary

computation,Honolulu,usa,IEEE, 17331738,2002

12. W.E.Hart,T.EKammeyer and R.K.Belew, the role of development in $\mathrm{GA}, 3^{\text {rd }}$ workshop on foundation of GA,sanfransico,page 315332,1995

13. JA Jones,MGKay, R KING and C Culbert, a hybrid GA for manufacturing cell design, journal of the Chinese institute of industrial engineering,vol 17,page 549-564,2000

14. T.EI Mihoub,AHogood,LNolle and A Battersby, performance of hybrid GAincorporating local search, in $18^{\text {th }}$ European simulation muticonference,

Horton,Magdeburg,Germany, 154-160,2004

15. T.Sasaki and $M$ Tokoro, adaptation toward chaning environments, why Darwinian in nature?, fourth European conference on artificial life,complex adaptive systems series,P Husbands and I Harvey,brighton UK MIT press 1997

16. H.Ishibuchi, SKaige, and $\mathrm{K}$ Narukawa, comparis on between Lamarckian and baldwinian repair on multiobjective o/1 knapsack problems, in evolutionary multi criterion optimization, carlos A coellocoello,A H Agurre and EZitzler, edsguanajjuato, mexico,2005

17. G Magyar, M Johnson, and O Nevalainen, an adaptive hybrid GA for the Press matching problem, IEEE transaction on evolutionary computation, vol 4,2000

18. F BEspinoza,BMinsker, D Goldberg, a elf adaptive hybrid GA in the genetic evolutionary computation

conference,s anfrancis co,morgankaufmann publis gers, 2001

19. D E Goldberg and S Voessner, optimization global local search hybrids in the genetic evolutionary computation conference Orlando usa,1999

20. W E Hart,CRRosin,RKBelew, GM Morris , improved evolutionary hybrids for flexible ligand docking in the autodock in optimization in computational chemistry and molecular biology,C A Floudas and PM Pardalos,springer 2000

21. H.Ishibuchi, T, Yoshidanad T Murata, balance between genetic search and local search in memt ic algorith $\mathrm{m}$ for multiobjective permutation flow shop scheduling, IEEE transaction on evolutionary computation vol 7,2003

22. B Ju istron,comparingdarwan inian, baldawinian and lamarckinian search in a genetic algorithm for the 4 cycle problem genetic evolutionary conference 1999 
23. KA Hacker, J Eddy ,ke Lewis, efficient global optimization using hybrid genetic algorithms, presented $\mathrm{t} 9^{\text {th }}$ AIAA-ISSMO symposium on multidisciplinary analysis and optimization, Atlanta USA, 2002

24. M.Land, evolutionary algorithms with local search for combinatorial optimization, doctoral dissertation, san diego university of California, 1998

25. LT Leng, guided GA, doctoral dissertation, university of Es sex,1999

26. EP Tsang and C Voudouris, fast local search and guided local search and their application to British telecom's workforce scheduling problem, in operations research letters, vol 20,1997

27. C Houck, ,JJones,M Kay, J Wilson, empirical investigation of the benefits of partial lamarckianis m , evolutionary computation vol5, 1997

28. Turney, myths and legends of the Baldwin effects, in workshop on evolutionary computation and machine learning at $13^{\text {th }}$ international conference on the machine learning Bari 1996

29. Z Michalweiz, G Nazhiyath, genocop 3, a coevolutionary algorithm for numerical optimxation problem with nonlinear constraints, $2^{\text {nd }}$ IEEE international conference on evolutionary computing vol 2, perth Australia, IEEE 1995

30. K Mathais,L Whitney, genetic operators the fitness landscape and the traveling salesman problem, in parallel solving form nature, ,Belgiu m,1992

31. T El Mihoub, Ahopgood,LNolle, A Battersby, a self-adaptive baldiwanian search in hybrid GA ,springer 2006

32. F Golver,tabu search part 1,ORSA Journal on computing vol 1, 1989

33. N Krasnogar and J Smith, emergence of profitable search strategies based on a simple inheritance mechanism, in genetic evolutionary computation conference, San Francisco USA, 2001 